arXiv:0811.2305 [hep-th]

WU-AP/294/08

\title{
Black Hole-Black String Phase Transitions FROM HYDRODYNAMICS
}

\author{
Kei-ichi Maeda* and Umpei Miyamoto ${ }^{\dagger}$ \\ * Department of Physics, Waseda University, Okubo 3-4-1, Tokyo 169-8555, Japan \\ ${ }^{\dagger}$ Racah Institute of Physics, Hebrew University, Givat Ram, Jerusalem 91904, Israel \\ maeda@waseda.jp umpei@phys.huji.ac.il
}

\begin{abstract}
We discuss the phase transitions between three states of a plasma fluid (plasma ball, uniform plasma tube, and non-uniform plasma tube), which are dual to the corresponding finite energy black objects (black hole, uniform black string, and non-uniform black string) localized in an asymptotically locally AdS space. Adopting the equation of state for the fluid obtained by the Scherk-Schwarz compactification of a conformal field theory, we obtain axisymmetric static equilibrium states of the plasma fluid and draw the phase diagrams with their thermodynamical quantities. By use of the fluid/gravity correspondence, we predict the phase diagrams of the AdS black holes and strings on the gravity side. The thermodynamic phase diagrams of the AdS black holes and strings show many similarities to those of the black hole-black string system in a Kaluza-Klein vacuum. For instance, the critical dimension for the smooth transition from the uniform to non-uniform strings is the same as that in the Kaluza-Klein vacuum in the canonical ensemble. The analysis in this paper may provide a holographic understanding of the relation between the Rayleigh-Plateau and Gregory-Laflamme instabilities via the fluid/gravity correspondence.
\end{abstract}




\section{Contents}

1 Introduction 1

2 Hydrostatics of Axisymmetric Plasma Lumps 3

2.1 Relativistic Navier-Stokes Equations . . . . . . . . . . . . . . . . . . 3

2.2 The Equivalent Plateau Problem and Rayleigh-Plateau Instability . . . . . . . . . . . 5

3 Equation of State and Thermodynamic Variables $\quad 7$

3.1 Equation of State . . . . . . . . . . . . . . . . . . . . 7

3.2 Uniform Tube and Spherical Ball Phases . . . . . . . . . . . . . . . . . 8

3.3 Non-uniform Tube Phase . . . . . . . . . . . . . . . . . . . . . . . . . . 10

4 Phase Structure $\quad 11$

4.1 Microcanonical Ensemble: $\hat{S}=\hat{S}(\hat{E}) \ldots \ldots \ldots \ldots \ldots \ldots \ldots$

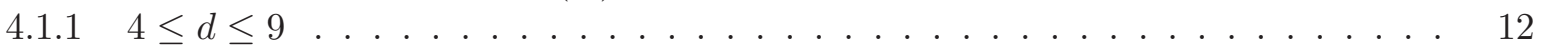

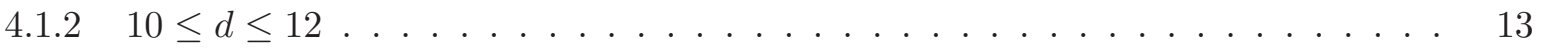

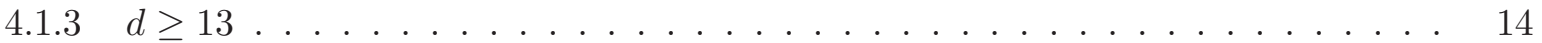

4.2 Canonical Ensemble: $\hat{F}=\hat{F}(\hat{T}) \ldots \ldots \ldots \ldots \ldots \ldots \ldots$

5 Summary and Discussion $\quad 16$

$\begin{array}{ll}\text { A Manipulations for Accurate Numerics } & 17\end{array}$

$\begin{array}{lr}\text { B Justification and Interpretation of Cusp Structures } & 18\end{array}$

\section{Introduction}

The AdS/CFT correspondence provides one of the best ways to explore the nonperturbative regimes of a certain class of strongly coupled quantum field theories by working in classical gravity. Conversely, from the viewpoint of black hole physics, we may be able to understand strong gravitational phenomena by studying an appropriate limit of field theories. In this paper, we investigate a class of AdS black holes, in particular, their phase structures and possible phase transitions, via the fluid/gravity correspondence (see, e.g., $[1,2]$ ), which has been proposed as a generalization of the AdS/CFT correspondence.

Recently, Aharony, Minwalla, and Wiseman [3] argued that in a class of large- $N$ gauge theories, a plasma ball (i.e., a lump of 'gluon' plasma) appears via a first-order deconfinement phase transition above a critical temperature. They also claimed that such a plasma ball will map to a finite energy black hole localized in the IR (infrared) region, and discussed what happens on the gravity side by studying various phenomena on the fluid side such as a plasma ball production and its subsequent decay by hadronization. In particular, they explicitly constructed a numerical domain wall solution to the Einstein equations with a negative cosmological constant which smoothly interpolates two spacetimes corresponding to the confined and deconfined phases. As a generalization of the analysis in [3], Lahiri and Minwalla [4] constructed a rotating plasma ring and a ball, which are dual to a rotating black ring and a rotating black hole, respectively (see also [5]). By construction (which we will briefly review in the text), these gravity duals reside in the IR region of AdS space compactified on a Scherk-Schwarz circle. This kind of black ring and hole solutions have not been discovered yet. 
However, by comparing the phase diagrams of a plasma ring and plasma ball with those of a black ring and black hole in an asymptotically flat space, they found qualitative agreements between them.

In this paper, we investigate the phase structures of another important class of black objects, i.e., black strings and black holes in a spacetime in which some spatial dimensions are compactified, from the viewpoint of the fluid/gravity correspondence (see [6] for the analysis on a similar system from a holographic viewpoint). We solve the $d$-dimensional relativistic Navier-Stokes equations [2] with an appropriate surface term to obtain static axisymmetric fluid equilibrium configurations. The configurations contain a spherical ball, a uniform tube, and a non-uniform tube. To see what their gravity duals are, we recall the arguments in [3]. We consider the following solution to the $(d+2)$ dimensional Einstein equations with a negative cosmological constant, $R_{a b}=-(d+1) \ell^{-2} g_{a b}$ :

$$
d s_{d+2}^{2}=\ell^{2}\left(e^{2 u}\left[-d t^{2}+T_{2 \pi}(u) d \theta^{2}+d w_{i}^{2}\right]+\frac{1}{T_{2 \pi}(u)} d u^{2}\right),
$$

where $i=1,2, \ldots, d-1$, and $\theta \in[0,2 \pi)$. The function $T_{x}(u)$ is defined by

$$
T_{x}(u)=1-\left[\frac{x}{4 \pi}(d+1) e^{u}\right]^{-(d+1)} .
$$

This spacetime is regarded as an $\mathrm{AdS}_{d+2}$ space with a Scherk-Schwarz compactification, which is called the AdS soliton [7]. By taking $u \rightarrow \infty$, one recovers the $\mathrm{AdS}_{d+2}$ in the Poincaré coordinates with a uniform circle of $\theta$. It should be noted that there is a cutoff in the IR region (i.e., small- $u$ region) since the Scherk-Schwarz circle shrinks to a point at a finite value of $u$. Imposing a periodicity in the imaginary time, i.e., $\tau \equiv \tau+\beta,(\tau=i t)$, one can regard this spacetime as a thermal gas of gravitons at temperature $T=\beta^{-1}$. In addition, there exists another exact solution

$$
d s_{d+2}^{2}=\ell^{2}\left(e^{2 u}\left[-T_{\beta}(u) d t^{2}+d \theta^{2}+d w_{i}^{2}\right]+\frac{1}{T_{\beta}(u)} d u^{2}\right)
$$

which has the same asymptotics as the spacetime (1). This solution can be regarded as a black brane with temperature $T=\beta^{-1}$. Considering both spacetimes, Eqs. (1) and (3), together in an ensemble, one can show that in the low temperature regime $\left(T<T_{c}=1 / 2 \pi\right)$ the AdS soliton (1) has a lower free energy, while in the high temperature regime $\left(T>T_{c}\right)$ the black brane (3) has a lower free energy and dominates. Thus, the system undergoes a Hawking-Page type phase transition at $T=T_{c}[7,8]$.

In $[3,4]$, the plasma balls and plasma rings which extend uniformly in the $\theta$-direction and nonuniformly in the $w_{i}$-directions (which are assumed to be noncompact) were constructed on the UV (ultraviolet) boundary by solving the fluid equations ${ }^{1}$. In this paper, we compactify one of $w_{i}$ 's on a circle, which we denote by coordinate $z$, and consider the plasma tube and plasma ball, where the former wraps the circle of the $z$-direction. As described in [4], the horizon topology of a dual black object is obtained by fibering the plasma configuration with an $S^{1}$ that shrinks to zero size at the fluid edges ${ }^{2}$. Therefore, the plasma ball $B^{d-1}$ and plasma tube $B^{d-2} \times S^{1}$, which we will obtain in this paper, map to the black objects whose horizon topologies are $S^{d}$ and $S^{d-1} \times S^{1}$, respectively. Namely, they are a black hole and a black string, respectively, in an asymptotically $\mathrm{AdS}_{d}$ space on the Scherk-Schwarz circle $S_{\theta}$ and Kaluza-Klein circle $S_{z}$.

\footnotetext{
${ }^{1}$ Note that the dual black holes and black rings also extend in the $u$-direction, although they are localized in this direction.

${ }^{2}$ This condition on the shrinking of the $S^{1}$ circle corresponds to the fact that at the IR wall mapped from the outside of the plasma, the Scherk-Schwarz circle shrinks to zero size.
} 
The gravity dual of the plasma tube obtained in this paper is rather different from the black string solutions in an asymptotically locally AdS space which have been studied extensively in the literature [9], as well as from the black strings in the asymptotically locally flat Kaluza-Klein space. We will see, however, that the thermodynamic properties of our AdS black hole-black string system display various qualitative similarities to those of the black hole-black string system in the asymptotically locally flat Kaluza-Klein space. The phase transitions and their dimensional dependence will be elaborated in this paper.

This paper is organized as follows. In Sec. 2, we reduce the Navier-Stokes equations for a plasma fluid to a hydrostatic equation for axisymmetric equilibrium states. We then demonstrate that this equation is equivalent to that of Plateau's problem (or capillary minimizing problem), obtaining the constant mean curvature surfaces which model soap bubbles with a variational principle. In addition, we show that the uniform plasma tube is unstable against a perturbation, which is the fluid counterpart [10-12] of the Gregory-Laflamme instability [13]. In Sec. 3, an equation of state for the fluid is introduced which is obtained from the Scherk-Schwarz compactification of a conformal field theory. The thermodynamic variables for each phase are then calculated. In Sec. 4, the thermodynamic phase structures are obtained. The final section is devoted to a summary and discussion. A technical issue about numerical integrations is treated in Appendix A. A justification of numerical results and a natural interpretation of phase diagram are described in Appendix B.

\section{Note Added}

During the preparation of this paper, we were informed that a similar work had been completed independently [14]. It is interesting to compare their results with ours.

\section{Hydrostatics of Axisymmetric Plasma Lumps}

\subsection{Relativistic Navier-Stokes Equations}

The equation of motion for the boundary fluid is simply given by the conservation of the stress tensor [2],

$$
\nabla_{\mu} T^{\mu \nu}=0
$$

The stress tensor consists of the perfect fluid part, the dissipative part, and the surface contribution in a certain long wavelength limit as

$$
T^{\mu \nu}=T_{\text {perfect }}^{\mu \nu}+T_{\text {surface }}^{\mu \nu}+T_{\text {dissipative }}^{\mu \nu},
$$

where the perfect fluid part takes the usual form as

$$
T_{\text {perfect }}^{\mu \nu}=(\rho+P) u^{\mu} u^{\mu}+P g^{\mu \nu}
$$

with the pressure $P$, proper energy density $\rho$, and velocity field $u^{\mu}$. The surface contribution can be written as

$$
T_{\text {surface }}^{\mu \nu}=\sigma\left(n^{\mu} n^{\nu}-g^{\mu \nu}\right) \sqrt{\partial \Phi \cdot \partial \Phi} \delta(\Phi)
$$

with $\sigma$ being a tension of the boundary. We have assumed that the surface of the fluid is given by

$\Phi\left(x^{\mu}\right)=0$ and the unit normal of this surface is denoted by $n_{\mu}:=\partial_{\mu} \Phi(\partial \Phi \cdot \partial \Phi)^{-1 / 2}$. For static 


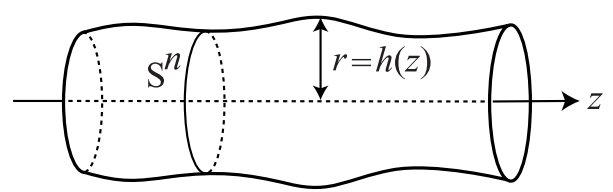

Figure 1: An axisymmetric static equilibrium state of fluid in a $d=(n+3)$-dimensional flat spacetime schematically embedded in a three dimensional space.

fluids which we will study in this paper one can show the dissipative term does not contribute to the equation of motion.

Now, we consider a $d=(n+3)$-dimensional flat spacetime $(n \geq 1)^{3}$ in cylindrical coordinates $x^{\mu}=\left(t, z, r, \phi^{i}\right),(i=1,2, \cdots, n)$,

$$
\begin{aligned}
g_{\mu \nu} \mathrm{d} x^{\mu} \mathrm{d} x^{\nu} & =-\mathrm{d} t^{2}+\mathrm{d} z^{2}+\mathrm{d} r^{2}+r^{2} \gamma_{i j}(\phi) \mathrm{d} \phi^{i} \mathrm{~d} \phi^{j} \\
& =\eta_{a b} \mathrm{~d} x^{a} \mathrm{~d} x^{b}+r^{2} \gamma_{i j}(\phi) \mathrm{d} \phi^{i} \mathrm{~d} \phi^{j}
\end{aligned}
$$

where $x^{a}=(t, z, r), \eta_{a b}=$ diag. $(-1,1,1)$ and $\gamma_{i j} \mathrm{~d} \phi^{i} \mathrm{~d} \phi^{j}$ is the line element of the unit $n$-sphere. Non-zero components of the Christoffel symbol are

$$
\Gamma_{i j}^{a}=-r \delta_{r}^{a} \gamma_{i j}, \quad \Gamma_{j a}^{i}=r^{-1} \delta_{a}^{r} \delta_{j}^{i}, \quad \Gamma_{j k}^{i}=\bar{\Gamma}^{i}{ }_{j k},
$$

where the bar is used for the variables with respect to the metric $\gamma_{i j}$. Assuming that the fluid is static in this frame $\left(u^{\mu}=\delta_{t}^{\mu}\right)$, the perfect fluid part of the stress tensor and its divergence are given by

$$
T_{\text {perfect }}^{\mu \nu}=\left(\begin{array}{cccc}
\rho & 0 & 0 & 0 \\
0 & P & 0 & 0 \\
0 & 0 & P & 0 \\
0 & 0 & 0 & r^{-2} P \gamma^{i j}
\end{array}\right), \quad \nabla_{\mu} T_{\text {perfect }}^{\mu \nu}=\left(0, \frac{\partial P}{\partial z}, \frac{\partial P}{\partial r}, 0\right),
$$

where we have used an identity $\bar{\nabla}_{i} \gamma_{j k} \equiv 0$. The boundary surface of an axisymmetric fluid configuration can be given by $\Phi(z, r):=r-h(z)=0$, where $h(z)$ is a height function (see Fig. 1) assumed to be a single-valued function. Then, noting that the unit normal vector $n^{\mu}$ is given by

$$
n^{\mu}=\frac{1}{\sqrt{1+h^{\prime 2}}}\left(\delta_{r}^{\mu}-h^{\prime} \delta_{z}^{\mu}\right), \quad h^{\prime}:=\partial_{z} h
$$

the surface contribution to the stress tensor and its divergence are given by

$$
\begin{aligned}
& T_{\text {surface }}^{\mu \nu}=\frac{\sigma \delta(r-h)}{\sqrt{1+h^{\prime 2}}}\left(\begin{array}{cccc}
1+h^{\prime 2} & 0 & 0 & 0 \\
0 & -1 & -h^{\prime} & 0 \\
0 & -h^{\prime} & -h^{\prime 2} & 0 \\
0 & 0 & 0 & -r^{-2}\left(1+h^{\prime 2}\right) \gamma^{i j}
\end{array}\right) \\
& \nabla_{\mu} T_{\text {surface }}^{\mu \nu}=\sigma \delta(r-h)\left(0,-(n+1) h^{\prime} H,(n+1) H, 0\right) .
\end{aligned}
$$

\footnotetext{
${ }^{3}$ In fact, the conformal boundary, where the plasma fluid resides, is $(d+1)$-dimensional if we take into account the existence of the Scherk-Schwarz circle, in which direction we assume the fluid configuration is uniform. Therefore, the total spacetime dimension of the AdS bulk is $d+2$.
} 
Here, $H$ is the mean curvature of the surface defined by

$$
H(z):=\frac{1}{n+1}\left(-\frac{h^{\prime \prime}}{\left(1+h^{\prime 2}\right)^{3 / 2}}+\frac{n}{h \sqrt{1+h^{\prime 2}}}\right) .
$$

The first and second terms in the parenthesis correspond to the axial and azimuthal principal curvatures of the surface, respectively (see, e.g., [12]).

The non-trivial components of Eq. (4) are

$$
\begin{aligned}
& \frac{\partial P}{\partial z}-(n+1) \sigma \delta(r-h) h^{\prime} H=0 \\
& \frac{\partial P}{\partial r}+(n+1) \sigma \delta(r-h) H=0 .
\end{aligned}
$$

Away from the boundary $r=h(z)$, we have the equation in the bulk, which gives

$$
P(z, r)=\text { const. }
$$

Integrating the equations of motion across the boundary, we have

$$
P_{>}-P_{<}+(n+1) \sigma H(z)=0
$$

where $P_{>}$and $P_{<}$are the pressures just outside and inside the boundary, respectively. Now, we consider the case in which the pressure outside vanishes $\left(P_{>}=0\right)$ for simplicity. Then, Eq. (16) reads

$$
\frac{1}{n+1}\left(-\frac{h^{\prime \prime}}{\left(1+h^{\prime 2}\right)^{3 / 2}}+\frac{n}{h \sqrt{1+h^{\prime 2}}}\right)=\frac{P_{<}}{(n+1) \sigma}=: H_{0}
$$

This is the governing equation that determines the axisymmetric equilibrium states of the fluid. This equation implies that the fluid surface is given by a constant mean curvature surface. As we will see, this equation is derived by a variational principle which minimizes the surface area of the fluid while keeping the volume fixed. A uniform tube found as a trivial solution of Eq. (17) will suffer from the Rayleigh-Plateau instability ${ }^{4}$.

\subsection{The Equivalent Plateau Problem and Rayleigh-Plateau Instability}

In general, the interior volume and surface area of a fluid body are written as

$$
V=\int_{\text {interior }} \mathrm{d} V, \quad A=\int_{\text {surface }} \mathrm{d} A .
$$

When we assume the staticity and axisymmetry of the fluid of which surface is given by $r=h(z)$, the induced metric on the surface is given by

$$
\mathrm{d} s_{\text {surface }}^{2}=-\mathrm{d} t^{2}+\left(1+h^{\prime 2}\right) \mathrm{d} z^{2}+h^{2} \gamma_{i j}(\phi) \mathrm{d} \phi^{i} \mathrm{~d} \phi^{j} .
$$

\footnotetext{
${ }^{4}$ The axisymmetric constant mean curvature surfaces in general dimensions were obtained in [11], and their geometric properties were investigated to compare those of black strings.
} 
Now, we assume that the $z$-direction is compactified on a circle, $z \in[-L / 2, L / 2]^{5}$. Noting that the volume and surface elements in Eq. (18) are given by $\mathrm{d} V=r^{n} \sqrt{\gamma} \mathrm{d} r \wedge \mathrm{d} z \wedge \mathrm{d} \phi^{1} \wedge \cdots \wedge \mathrm{d} \phi^{n}$ and $\mathrm{d} A=h^{n} \sqrt{\left(1+h^{\prime 2}\right) \gamma} \mathrm{d} z \wedge \mathrm{d} \phi^{1} \wedge \cdots \wedge \mathrm{d} \phi^{n}$, we have

$$
\begin{aligned}
& V[h]=\Omega_{n} \int_{-L / 2}^{L / 2} \mathrm{~d} z h^{n+1}(z), \quad A[h]=(n+1) \Omega_{n} \int_{-L / 2}^{L / 2} \mathrm{~d} z \sqrt{1+h^{\prime 2}} h^{n}(z), \\
& \Omega_{n}:=\frac{1}{n+1} \int_{S^{n}} \sqrt{\gamma} \mathrm{d} \phi^{1} \wedge \cdots \wedge \mathrm{d} \phi^{n}=\frac{\pi^{(n+1) / 2}}{\Gamma[(n+1) / 2+1]} .
\end{aligned}
$$

The equation of motion for the configuration that minimizes (or extremalizes) the surface area for a given volume is obtained by varying the action

$$
I[h]:=\sigma_{0} A[h]-p_{0} V[h] .
$$

Here, the constant $\sigma_{0}$ corresponds to the surface tension, while the constant $p_{0}$ corresponds to the pressure, but it is just a Lagrange multiplier to mathematically ensure that the volume is fixed. The Euler-Lagrange equation obtained by variation $\left(\delta_{h} I=0\right)$ is equivalent to Eq. (17) with the identification of $p_{0} \leftrightarrow P_{<}$and $\sigma_{0} \leftrightarrow \sigma$.

It is noted that by adding a term corresponding to the rotation energy to the action (21), which is roughly given by $-\omega J$ with an angular velocity $\omega$ and an angular momentum $J$, the equation of $h(z)$ for a rigidly rotating non-relativistic fluid is obtained. In the rotational case, the surface deviates from a constant mean curvature surface $[4,5,10]^{6}$

Equation (17) has two trivial solutions representing a uniform tube (UT) and a spherical ball (SB),

$$
\begin{array}{ll}
h=h_{\mathrm{UT}}:=r_{0}, & H=H_{\mathrm{UT}}:=\frac{n}{(n+1) r_{0}}, \\
h=h_{\mathrm{SB}}:=\sqrt{R_{0}^{2}-z^{2}}, & H=H_{\mathrm{SB}}:=\frac{1}{R_{0}} .
\end{array}
$$

As is well known, a translationally invariant cylindrical body is unstable if the linear dimension is longer than its circumference in $d=4$ (the Rayleigh-Plateau instability). Its onset mode in general dimensions is obtained by the following static perturbation of the uniform tube [10,11]. First, we expand $h(z)$ around the uniform tube,

$$
h(z)=r_{0}+\varepsilon h_{1}(z)+O\left(\varepsilon^{2}\right) .
$$

\footnotetext{
${ }^{5}$ This compactification corresponds to compactifying a spatial direction of the field theory coordinates in the Poincaré patch from the AdS point of view. However, it does not introduce the problem of singularities in the asymptotic IR region since the IR is cutoff by the horizon or the shrinking of the Scherk-Schwarz circle. We would like to thank T. Wiseman for discussion on this point.

${ }^{6}$ See Ref. [14] for more general relations between the equation of motion (16), called the Young-Laplace equation, and variational principles. They showed that in a fully covariant way the Young-Laplace equation can be obtained by variational principle of either the entropy maximization or potential energy minimization. It was also shown that these two variational principles reduce to the area minimization with volume fixing in the static situation. Its special case corresponds to ours. Their general proofs nicely relate the Young-Laplace equation (derived from the Navier-Stokes equation), thermodynamic equilibrium condition (the entropy maximization), mechanical equilibrium condition (the potential energy minimization), geometrical condition (the area minimization). More importantly it also supports the original argument in [3] (and our thermodynamic consideration in Sec. 4), that the thermodynamics of finite energy black holes in the Scherk-Schwarz compactified AdS is mapped holographically to that of deconfined plasma lumps held by surface tension. The authors thank an anonymous referee for letting us know this point.
} 
Substituting this expansion into Eq. (17), we have the linear perturbation equation at $O(\varepsilon)$,

$$
h_{1}^{\prime \prime}+\frac{(n+1)^{2} H_{\mathrm{UT}}^{2}}{n} h_{1}=0 .
$$

With a boundary condition, say $h_{1}^{\prime}(0)=0$, we have

$$
h_{1}(z)=h^{(1)} \cos \left(k_{\mathrm{RP}} z\right), \quad k_{\mathrm{RP}}:=\frac{\sqrt{n}}{r_{0}},
$$

where $h^{(1)}$ is an integration constant. Solution (25) corresponds to the marginally stable mode of the Rayleigh-Plateau instability. The uniform tube is unstable if the length of the cylinder $L$ satisfies $L>L_{\mathrm{RP}}:=2 \pi / k_{\mathrm{RP}}$. In other words, the uniform tube is unstable if the radius $r_{0}$ satisfies $r_{0}<$ $r_{\mathrm{RP}}:=\sqrt{n} L / 2 \pi$ for a given $L$. This dimensional dependence of the critical mode is quite similar to that of the Gregory-Laflamme instability. See Refs. [10-12] for more on the similarities between the Rayleigh-Plateau and Gregory-Laflamme instabilities.

\section{Equation of State and Thermodynamic Variables}

In order to investigate the thermodynamic properties of the fluid lumps in the context of AdS/CFT, we introduce an equation of state for the plasma fluid. Then, the thermodynamic variables for each phase of fluid lumps are calculated.

\subsection{Equation of State}

The equation of state for the plasma is obtained by the Scherk-Schwarz compactification of a $(d+1)$ dimensional conformal field theory [3]. The free energy in terms of temperature $T$ and volume $\mathcal{V}$ is given by

$$
\mathcal{F}=\left(\rho_{0}-\alpha T^{d+1}\right) \mathcal{V}
$$

where $\rho_{0}$ is a vacuum energy and $\alpha$ is some constant. The pressure $P$, entropy $\mathcal{S}$, and energy $\mathcal{E}$ are given by

$$
\begin{aligned}
& P=-\left(\frac{\partial \mathcal{F}}{\partial \mathcal{V}}\right)_{T}=-\rho_{0}+\alpha T^{d+1}, \\
& \mathcal{S}=-\left(\frac{\partial \mathcal{F}}{\partial T}\right)_{\mathcal{V}}=(d+1) \alpha \mathcal{V} T^{d}, \\
& \mathcal{E}:=\mathcal{F}+T \mathcal{S}=\left(\rho_{0}+d \alpha T^{d+1}\right) \mathcal{V} .
\end{aligned}
$$

We then introduce the energy density $\rho$ and entropy density $s$, given as

$$
\begin{aligned}
& \rho:=\frac{\mathcal{E}}{\mathcal{V}}=\rho_{0}+d \alpha T^{d+1}, \\
& s:=\frac{\mathcal{S}}{\mathcal{V}}=(d+1) \alpha^{1 /(d+1)}\left(\rho_{0}+P\right)^{d /(d+1)} .
\end{aligned}
$$


From the parameters $\rho_{0}, \sigma$, and $\alpha$, a characteristic length scale (whose existence violates the conformal invariance), temperature, and entropy density can be defined as

$$
l_{0}:=\frac{\sigma}{\rho_{0}}, \quad T_{c}:=\left(\frac{\rho_{0}}{\alpha}\right)^{1 /(d+1)}, \quad s_{0}:=\left(\alpha \rho_{0}^{d}\right)^{1 /(d+1)} .
$$

The temperature in Eq. (29) is nothing but the critical temperature of the confine-deconfine phase transition which occurs at $f:=\mathcal{F} / \mathcal{V}=-P=0$.

Now, we consider the thermodynamic variables for the plasma lumps, that is, not only those for their constituent plasma but also the contributions from the surface term. Their energy density and entropy density are given by

$$
\begin{aligned}
& T^{t t}=\rho+\sigma \delta(r-h) \sqrt{1+h^{\prime 2}}, \\
& s=(d+1) s_{0}\left(\frac{T}{T_{c}}\right)^{d} .
\end{aligned}
$$

The energy, entropy, and Helmholtz free energy for the plasma lumps can be obtained by integrating the above densities,

$$
\begin{aligned}
& E=\int\left(T_{\text {perfect }}^{t t}+T_{\text {surface }}^{t t}\right) \mathrm{d} V=\rho V+\sigma A, \\
& S=\int s \mathrm{~d} V=s V, \\
& F=E-T S=-P V+\sigma A,
\end{aligned}
$$

where we have used that the energy density and entropy density are constant in the present system.

From the relations of $P=-\rho_{0}+\alpha T^{n+4}$ and $P=(n+1) \sigma H$ (note that $d=n+3$ ), we obtain a relation between the temperature $T$ and mean curvature $H$,

$$
T=T_{c}\left[1+(n+1) \tilde{l}_{0} H L\right]^{1 /(n+4)}, \quad \tilde{l}_{0}:=\frac{l_{0}}{L} .
$$

We will discuss later a physically reasonable choice of the dimensionless parameter $\tilde{l}_{0}$.

It is convenient to define the following normalized dimensionless variables when we draw phase diagrams,

$$
\hat{E}:=\frac{E}{E_{\mathrm{RP}}}, \quad \hat{S}:=\frac{S}{S_{\mathrm{SB}}}, \quad \hat{F}:=\frac{F}{F_{\mathrm{SB}}}, \quad \hat{T}:=\frac{T}{T_{\mathrm{RP}}},
$$

where $E_{\mathrm{RP}}$ and $T_{\mathrm{RP}}$ are the energy and the temperature of the critical uniform tube, and $S_{\mathrm{SB}}$ and $F_{\mathrm{SB}}$ are the entropy and free energy of the spherical ball, respectively, which are given in the following subsection.

\subsection{Uniform Tube and Spherical Ball Phases}

For the uniform tube, $h_{\mathrm{UT}}(z)=r_{0}$ with the length $L$, we can obtain the thermodynamic variables as functions of single parameter $r_{0} / L$,

$$
\begin{aligned}
& E_{\mathrm{UT}}=\rho_{0} \frac{\Omega_{n} L^{n+2}}{n}\left[\left(n^{2}+4 n+1\right) \tilde{T}_{\mathrm{UT}}^{n+4}-1\right]\left(\frac{r_{0}}{L}\right)^{n+1}, \quad S_{\mathrm{UT}}=s_{0}(n+4) \Omega_{n} L^{n+2} \tilde{T}_{\mathrm{UT}}^{n+3}\left(\frac{r_{0}}{L}\right)^{n+1}, \\
& F_{\mathrm{UT}}=\rho_{0} \frac{\Omega_{n} L^{n+2}}{n}\left(\tilde{T}_{\mathrm{UT}}^{n+4}-1\right)\left(\frac{r_{0}}{L}\right)^{n+1}, \quad T_{\mathrm{UT}}=T_{c}\left[1+n \tilde{l}_{0}\left(\frac{L}{r_{0}}\right)\right]^{1 /(n+4)},
\end{aligned}
$$


where $\tilde{T}_{\mathrm{UT}}:=T_{\mathrm{UT}} / T_{c}$. The critical values for the RP instability are obtained just by setting $r_{0}=r_{\mathrm{RP}}$ $(=\sqrt{n} L / 2 \pi)$ in Eq. (34). For instance, the energy and temperature of the critical tube are given by

$$
E_{\mathrm{RP}}=\rho_{0} \frac{\Omega_{n} L^{n+2}}{n}\left(\frac{\sqrt{n}}{2 \pi}\right)^{n+1}\left[\left(n^{2}+4 n+1\right) \tilde{T}_{\mathrm{RP}}^{n+4}-1\right], \quad T_{\mathrm{RP}}=T_{c}\left(1+2 \pi \sqrt{n} \tilde{l}_{0}\right)^{1 /(n+4)},
$$

where $\tilde{T}_{\mathrm{RP}}:=T_{\mathrm{RP}} / T_{c}$.

For the spherical ball, $h_{\mathrm{SB}}(z)=\sqrt{R_{0}^{2}-z^{2}}$ in the period $L$, we have the thermodynamic variables as functions of a single parameter $R_{0} / L$,

$$
\begin{aligned}
& E_{\mathrm{SB}}=\rho_{0} \frac{\Omega_{n+1} L^{n+2}}{n+1}\left[\left(n^{2}+5 n+5\right) \tilde{T}_{\mathrm{SB}}^{n+4}-1\right]\left(\frac{R_{0}}{L}\right)^{n+2}, \\
& S_{\mathrm{SB}}=s_{0}(n+4) \Omega_{n+1} L^{n+2} \tilde{T}_{\mathrm{SB}}^{n+3}\left(\frac{R_{0}}{L}\right)^{n+2}, \\
& F_{\mathrm{SB}}=\rho_{0} \frac{\Omega_{n+1} L^{n+2}}{n+1}\left(\tilde{T}_{\mathrm{SB}}^{n+4}-1\right)\left(\frac{R_{0}}{L}\right)^{n+2}, \quad T_{\mathrm{SB}}=T_{c}\left[1+(n+1) \tilde{l}_{0}\left(\frac{L}{R_{0}}\right)\right]^{1 /(n+4)},
\end{aligned}
$$

where $\tilde{T}_{\mathrm{SB}}:=T_{\mathrm{SB}} / T_{c}$.

According to the definition (33), we have the following dimensionless quantities,

$$
\begin{aligned}
& \hat{E}_{\mathrm{UT}}=\left(\frac{2 \pi}{\sqrt{n}}\right)^{n+1} \frac{\left(n^{2}+4 n+1\right)\left[1+n \tilde{l}_{0}\left(L / r_{0}\right)\right]-1}{\left(n^{2}+4 n+1\right)\left(1+2 \pi \sqrt{n} \tilde{l}_{0}\right)-1}\left(\frac{r_{0}}{L}\right)^{n+1}, \\
& \hat{S}_{\mathrm{UT}}=\frac{\Omega_{n}}{\Omega_{n+1}}\left[\frac{1+n \tilde{l}_{0}\left(L / r_{0}\right)}{1+(n+1) \tilde{l}_{0}\left(L / R_{0}\right)}\right]^{(n+3) /(n+4)}\left(\frac{r_{0}}{L}\right)^{n+1}\left(\frac{L}{R_{0}}\right)^{n+2}, \\
& \hat{F}_{\mathrm{UT}}=\frac{n^{n} \Omega_{n}}{(n+1)^{n+1} \Omega_{n+1}} \frac{\tilde{T}_{\mathrm{UT}}^{n+4}-1}{\tilde{l}_{0}}, \quad \hat{T}_{\mathrm{UT}}=\left[\frac{1+n \tilde{l}_{0}\left(L / r_{0}\right)}{1+2 \pi \sqrt{n} \tilde{l}_{0}}\right]^{1 /(n+4)}
\end{aligned}
$$

for the uniform tube phase, and

$$
\begin{aligned}
& \hat{E}_{\mathrm{SB}}=\frac{n \Omega_{n+1}}{(n+1) \Omega_{n}}\left(\frac{2 \pi}{\sqrt{n}}\right)^{n+1} \frac{\left(n^{2}+5 n+5\right)\left[1+(n+1) \tilde{l}_{0}\left(L / R_{0}\right)\right]-1}{\left(n^{2}+4 n+1\right)\left(1+2 \pi \sqrt{n} \tilde{l}_{0}\right)-1}\left(\frac{R_{0}}{L}\right)^{n+2}, \\
& \hat{T}_{\mathrm{SB}}=\left[\frac{1+(n+1) \tilde{l}_{0}\left(L / R_{0}\right)}{1+2 \pi \sqrt{n} \tilde{l}_{0}}\right]^{1 /(n+4)}, \quad \hat{S}_{\mathrm{SB}}=\hat{F}_{\mathrm{SB}} \equiv 1
\end{aligned}
$$

for the spherical ball phase.

Now, for each value of $\tilde{l}_{0}$, which should be specified, we obtain thermodynamic relations such as $\hat{S}=\hat{S}(\hat{E})$ and $\hat{F}=\hat{F}(\hat{T})$ implicitly via the parameter $r_{0} / L \in[0,+\infty)$ for the uniform tube phase and via $R_{0} / L \in[0,1 / 2]$ for the spherical ball phase, respectively ${ }^{7}$. Note that due to the fact $R_{0} \leq L / 2$ (i.e., the condition that a ball fits the period), the spherical ball phase has an upper bound of energy and a lower bound of the temperature.

\footnotetext{
${ }^{7}$ Because we normalize the entropy by that of the spherical ball, Eq. (33), the expression of $\hat{S}_{\mathrm{UT}}$ in Eq. (37) contains the radius of the spherical ball $R_{0}$. When one draws the $\left(\hat{E}_{\mathrm{UT}}, \hat{S}_{\mathrm{UT}}\right)$ curve by varying the parameter $r_{0}$, this $R_{0}$ should be regarded as a function of $r_{0}$. Such a relation $R_{0}=R_{0}\left(r_{0}\right)$ is obtained by the combination of $R_{0}=R_{0}(\hat{E})$ obtained by inversing the relation in Eq. (38) and $\hat{E}=\hat{E}\left(r_{0}\right)$ in Eq. (37). Similar transformations are needed to draw the $\left(\hat{E}_{\mathrm{NUT}}, \hat{S}_{\mathrm{NUT}}\right)$ curve [see $\hat{S}_{\mathrm{NUT}}$ in Eq. (43)].
} 


\subsection{Non-uniform Tube Phase}

As given in the preceding sections, Eq. (17) is solved by the uniform tube and the spherical ball. In addition to these two solutions, Eq. (17) allows the third solution, which is a non-trivial constant mean curvature surface corresponding to the non-uniform tube (NUT) [11]. For the $d=4$ case, this solution is known as Delaunay's unduloid [15], which is the surface of revolution of an elliptic catenary [see Fig. 2(a)]. Although a certain parametric representation of the curve is known for the $d=4$ case, for $d>4$, we have to integrate Eq. (17) numerically. As we will see below, if we introduce a 'non-uniformness parameter' $\lambda:=r_{-} / r_{+}$, where $r_{-}$and $r_{+}$are the smallest and largest radii of a non-uniform tube, all non-uniform tube solutions are described by a one-parameter family of $\lambda$, $(0<\lambda<1)$.

First, we introduce the following dimensionless variables,

$$
y:=H_{0} z, \quad w:=H_{0} h(z),
$$

where $H_{0}$ is the mean curvature of the non-uniform tube. Then, the first integral of Eq. (17) is written in a potential form,

$$
\left(\frac{\mathrm{d} w}{\mathrm{~d} y}\right)^{2}+U(w)=0, \quad U(w):=1-\left(\frac{w^{n}}{w^{n+1}+K}\right)^{2},
$$

where $K$ is an integration constant. We assume that there are two zero points of the potential $U(w)$ denoted by $w_{+}$and $w_{-}\left(0<w_{-}<w_{+}\right) . w_{+}$and $w_{-}$correspond to the maximum and minimum (dimensionless) radii of the non-uniform tube, respectively. Thus, $\lambda=w_{-} / w_{+} \rightarrow 0$ and $\lambda \rightarrow 1$ correspond to the ball and the critical tube, respectively [see Fig. 2(b) for a schematic picture of $U(w)]$. If we first give a $\lambda$, from $U\left(w_{ \pm}\right)=0$, we obtain

$$
w_{+}=\frac{1-\lambda^{n}}{1-\lambda^{n+1}}, \quad w_{-}=\frac{\lambda\left(1-\lambda^{n}\right)}{1-\lambda^{n+1}}, \quad K=\frac{(1-\lambda) \lambda^{n}\left(1-\lambda^{n}\right)^{n}}{\left(1-\lambda^{n+1}\right)^{n+1}} .
$$

Using $\mathrm{d} z=(\mathrm{d} z / \mathrm{d} h) \mathrm{d} h=\mathrm{d} w /\left(H_{0} \sqrt{-U}\right)$, one can express the period $L$, surface area $A$ and volume $V$

(a)

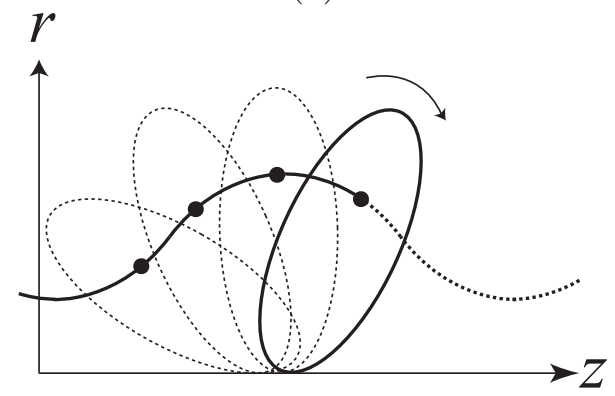

(b)

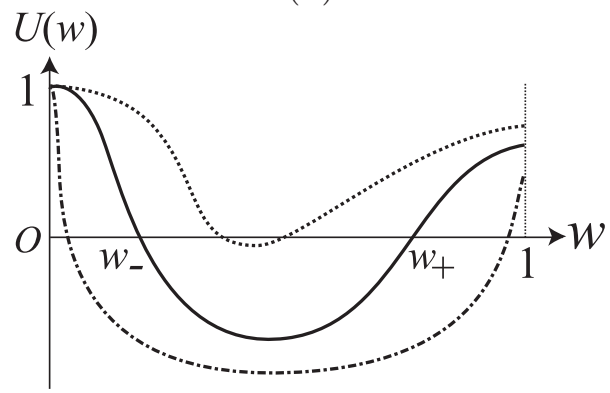

Figure 2: (a) The non-uniform tube in $d=4$ is called Delaunay's unduloid in geometry, which is the surface of revolution of an elliptic catenary. The elliptic catenary is obtained by rotating an ellipse along a line (i.e., the $z$-axis) and tracing the focus, marked by small solid circles. (b) A schematic picture of the potential $U(w)$, defined in Eq. (40), for three values of $\lambda=w_{-} / w_{+}$. From the top curve to the bottom, the value of $\lambda$ decreases within the range $0<\lambda<1$. 
per the period in terms of $\lambda$ and $H_{0}$,

$$
\begin{aligned}
& L_{\mathrm{NUT}}=\frac{2}{H_{0}} \int_{w_{-}}^{w_{+}} \mathrm{d} w \frac{1}{\sqrt{-U(w)}}=: \frac{1}{H_{0}} \tilde{L}(\lambda), \\
& A_{\mathrm{NUT}}=\frac{2(n+1) \Omega_{n}}{H_{0}^{n+1}} \int_{w_{-}}^{w_{+}} \mathrm{d} w w^{n} \sqrt{\frac{1-U(w)}{-U(w)}}=: \frac{(n+1) \Omega_{n}}{H_{0}^{n+1}} \tilde{A}(\lambda), \\
& V_{\mathrm{NUT}}=\frac{2 \Omega_{n}}{H_{0}^{n+2}} \int_{w_{-}}^{w_{+}} \mathrm{d} w \frac{w^{n+1}}{\sqrt{-U(w)}}=: \frac{\Omega_{n}}{H_{0}^{n+2}} \tilde{V}(\lambda) .
\end{aligned}
$$

As a result, for the non-uniform tube, we can obtain the thermodynamic dimensionless quantities as functions of the non-uniformness parameter $\lambda$,

$$
\begin{aligned}
\hat{E}_{\mathrm{NUT}} & =n\left(\frac{2 \pi}{\sqrt{n}}\right)^{n+1} \frac{[(n+3) \tilde{V}+\tilde{A}]\left[1+(n+1) \tilde{l}_{0} \tilde{L}\right]+\tilde{V}-\tilde{A}}{\left[\left(n^{2}+4 n+1\right)\left(1+2 \pi \sqrt{n} \tilde{l}_{0}\right)-1\right] \tilde{L}^{n+2}}, \\
\hat{S}_{\mathrm{NUT}} & =\frac{\Omega_{n}}{\Omega_{n+1}}\left[\frac{1+(n+1) \tilde{l}_{0} \tilde{L}}{1+(n+1) \tilde{l}_{0}\left(L / R_{0}\right)}\right]^{(n+3) /(n+4)} \frac{\tilde{V}}{\tilde{L}^{n+2}}\left(\frac{L}{R_{0}}\right)^{n+2}, \\
\hat{F}_{\mathrm{NUT}} & =\frac{n+1}{n^{n}} \frac{\left(\tilde{T}_{\mathrm{NUT}}^{n+4}-1\right)^{n}(\tilde{A}-\tilde{V})}{\tilde{l}_{0}^{n} \tilde{L}^{n+1}}, \\
\hat{T}_{\mathrm{NUT}} & =\frac{\Omega_{n}}{(n+1)^{n+1} \Omega_{n+1}}\left(\tilde{T}_{\mathrm{NUT}}^{n+4}-1\right)^{n+2} \frac{\tilde{A}-\tilde{V}}{\tilde{l}_{0}^{n+2} \tilde{L}^{n+2}},
\end{aligned}
$$

where $\tilde{T}_{\mathrm{NUT}}:=T_{\mathrm{NUT}} / T_{c}$, and $\tilde{L}(\lambda), \tilde{A}(\lambda)$, and $\tilde{V}(\lambda)$ are the dimensionless variables defined in Eq. (42). For each value of $\tilde{l}_{0}$, which should be specified, we have thermodynamic relations, $\hat{S}=\hat{S}(\hat{E})$ and $\hat{F}=\hat{F}(\hat{T})$, implicitly via the non-uniformness parameter $\lambda \in(0,1)$, (see also Footnote 7 ).

Now, we have expressed all thermodynamic variables as functions of the parameter $\lambda$ in the integration form. Although the integrations in Eq. (42) are of course finite, the integrands diverge at the both ends of the integration range since $U\left(w_{ \pm}\right)=0$. Therefore, one needs some manipulations for an accurate numerical integration, especially in the highly deformed regime $\lambda \ll 1$. We present some technical prescriptions making it possible to figure the fine structures of phase diagrams in Appendix A.

\section{Phase Structure}

In the previous section, we have described the necessary thermodynamic variables of each phase by the appropriate parameters: the uniform tube is parameterized by $r_{0} / L \in[0, \infty)$; the spherical ball by $R_{0} / L \in[0,1 / 2]$; and the non-uniform tube by $\lambda \in(0,1)$. To draw phase diagrams, we have to specify the undetermined parameter $\tilde{l}_{0}=l_{0} / L=\sigma /\left(\rho_{0} L\right)$. Although we are treating the sharp boundary as a fluid surface, in reality the boundary surface has a thickness of order $T_{c}^{-1} \sim \sigma / \rho_{0}=l_{0}[3,4]$. Therefore, we obviously should work in the limit $l_{0} \ll L$, where the thickness of the boundary can be ignored (except for highly deformed configurations). Otherwise higher-derivative contributions to the surface stress tensor would dominate. Hence, we take $\tilde{l}_{0}=1.0 \times 10^{-2}$ in all concrete examples hereafter. It should be stressed, however, that the qualitative aspects of the phase diagrams do not depend on a specific choices of parameter $\tilde{l}_{0}$. In particular, we have confirmed that the critical dimensions which we will find do not change for other choice of $\tilde{l}_{0}$ within the range of $1.0 \times 10^{-2} \leq \tilde{l}_{0} \lesssim 1$. It is noted 
that such a choice of small $\tilde{l}_{0}$ corresponds to $0<T_{\mathrm{RP}} / T_{c}-1 \ll 1$ as one can see from Eq. (35). That is, we only consider the plasma just above the critical temperature $T_{c}$.

\subsection{Microcanonical Ensemble: $\hat{S}=\hat{S}(\hat{E})$}

First we discuss the phase transitions among the plasma lumps in the microcanonical ensemble. Hence, we consider the $\hat{E}-\hat{S}$ relation. According to the behaviors of each phase curve, e.g., the number of cusps appearing in the $\hat{E}$ - $\hat{S}$ diagram here, we divide the dimensions into three groups, i.e., $4 \leq d \leq 9$, $10 \leq d \leq 12$, and $d \geq 13$. We now discuss the possible phase transitions between the three phases (a spherical ball, and uniform and non-uniform tubes) with the criterion that the maximum entropy state for a fixed energy is favored.

\subsection{1 $4 \leq d \leq 9$}

In this first class of dimensions, the branches of the uniform tube and spherical ball intersect at the point labeled by A in Fig. 3. The spherical ball always has a larger entropy than the critical tube.

From Fig. 3, one can see that the branch of non-uniform tubes emerges from the Rayleigh-Plateau critical point, located at $\hat{E}=1$, and reaches the end of the spherical ball branch located at the point $\mathrm{E}$, where the ball 'touches itself' at the boundary $z= \pm L / 2$. The most important point is that the non-uniform tube branch always has a smaller entropy than the other two. Thus, the phase diagram suggests that the non-uniform branch is never favored. Suppose that we have a fat uniform tube (i.e., large $\hat{E}$ ) and decrease its energy $\hat{E}$. As $\hat{E}$ decreases, the uniform branch meets the spherical ball branch at the point A, and at this point the uniform tube transits to a ball with a discontinuous jump in configuration. This transition is of first order.

Here, we can see that the energy-entropy diagram in $d=5$ is quite similar to the mass-entropy digram of the black hole-black string system in the asymptotically locally flat 5 and 6 dimensional Kaluza-Klein space (see Fig. 6 in [16]). Only one apparent difference is that the localized phase, i.e., the spherical plasma ball does not deform unlike a localized caged black hole.

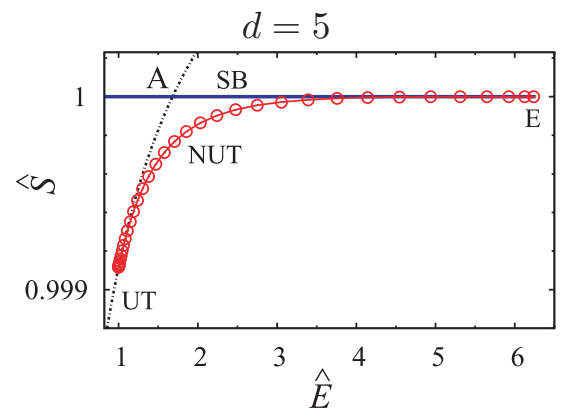

Figure 3: The energy-entropy diagram for $d=5$, containing the phases of the spherical ball (SB, bluesolid line), uniform tube (UT, black-dashed curve), and non-uniform tube (NUT, red-solid curve with small circles of data). The maximum entropy state for a given energy is favored. There is no cusp on the non-uniform tube phase, which is the case for $4 \leq d \leq 9$. 


\subsection{2 $10 \leq d \leq 12$}

In this class of dimensions, the phase diagrams are somewhat complicated due to the appearance of two cusps in the $(\hat{E}, \hat{S})$ diagram. The $(\hat{E}, \hat{S})$ diagrams for $d=10,11$ and 12 are shown in Fig. 4 . The uniform tube and spherical ball intersect in $d=10$ at the point $\mathrm{A}$, while they do not intersect any more in $d=11$ and 12 (in fact, ${ }^{\forall} d \geq 11$ ). The spherical ball has a larger entropy than the critical tube in $d=10$ and 11 , while this is not the case in $d=12$ (in fact, ${ }^{\forall} d \geq 12$ ). As can be seen from these observations, there appear rich and non-trivial phase structures in these transient dimensions.

From the fact that the non-uniform branch has two cusps in the $(\hat{E}, \hat{S})$ diagrams, the non-uniform tube branch can be divided into three branches (i.e., three smooth curves): the first branch leaves the Rayleigh-Plateau critical point; the second one is connected to the end point E of the spherical ball branch; the third one is the curve between the first and the second ones. The first and third branches correspond to the non-uniform tube branch appearing in the lower dimensions $(4 \leq d \leq 9)$. We shall first discuss the case of $d=11$. The non-uniform tube emerges from the Rayleigh-Plateau critical point. $\hat{E}$ increases at first, however, it turns to decrease, and then to increase again. Eventually the non-uniform tube branch reaches the end point $\mathrm{E}$ of the spherical ball branch. During this change, the non-uniform tube branch intersects with the uniform tube branch at the point $\mathrm{C}$ and with the spherical ball branch at the point B. Thus, if we begin with a fat uniform tube (i.e., large $\hat{E}$ ) and decreases $\hat{E}$, the uniform tube transits to the non-uniform tube at the point $\mathrm{C}$ with a discrete jump in configuration. As $\hat{E}$ decreases further, the non-uniform tube transits to a spherical ball at the point $\mathrm{B}$ with a discrete jump in configuration.

The phase structures of $d=12$ is quite similar to that in $d=11$. That is, if we begin with a fat uniform tube and decrease the energy, the favored phases shift as (uniform tube) $\rightarrow$ (non-uniform tube) $\rightarrow$ (spherical ball). These two phase transitions are accompanied by the discrete jumps in configuration, and hence they are of first order. However, the phase structure in $d=10$ is different from those of $d=11$ and $d=12$, although there are two cusps in all cases. The branches of uniform tube and spherical ball still intersects (at the point A). The spherical ball has a larger entropy than the critical uniform tube. As a consequence, the behavior of the non-uniform tube branch is similar to those in $4 \leq d \leq 9$ except that two quite small cusps appear at the middle of the branch. That is, if we begin with a fat uniform tube and decreases the energy, the uniform tube transits to the spherical ball at the point A. The non-uniform tube has nothing to do with this transition.
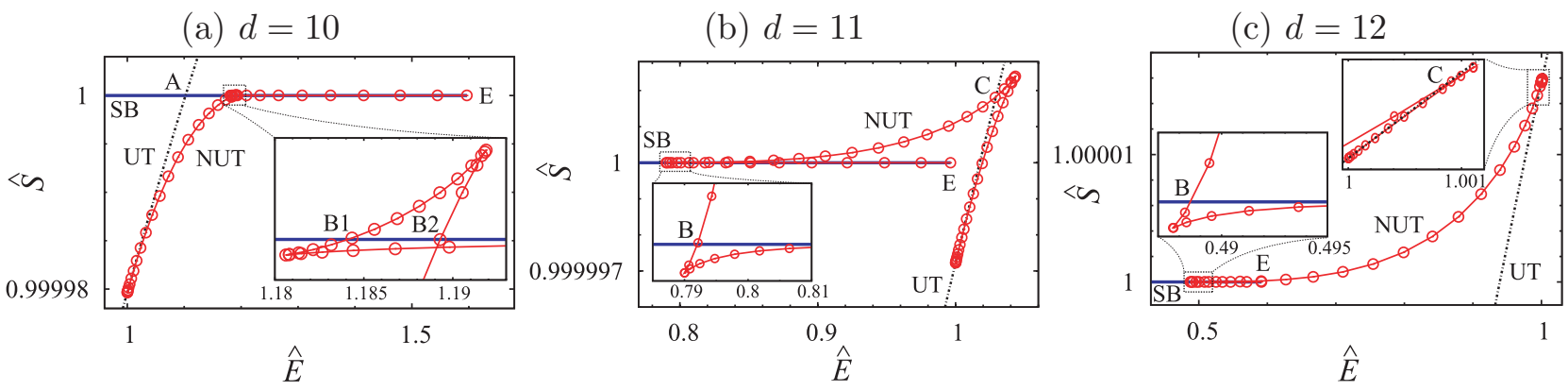

Figure 4: The energy-entropy diagrams for (a) $d=10$, (b) $d=11$, and (c) $d=12$. There appear two cusps on the non-uniform tube phase in this class of dimensions. 


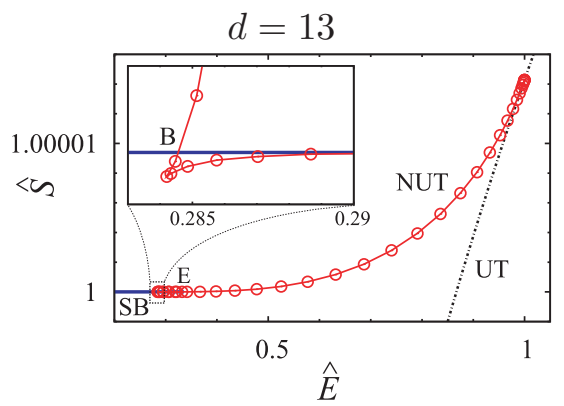

Figure 5: The energy-entropy diagram for $d=13$. There exists one cusp on the non-uniform tube phase, which is the case for $d \geq 13$.

\subsection{3 $d \geq 13$}

As mentioned before, the branches of the uniform tube and spherical ball do not intersect each other in this class of dimensions. The spherical ball phase has a smaller entropy than the critical uniform tube in this class of dimensions. The cusp near the Rayleigh-Plateau critical point disappears at $d=13$, while the cusp near the spherical ball phase remains (we have confirmed this up to $d=15$ ). Therefore, if we begin with a fat uniform tube (i.e., large $\hat{E}$ ) and decrease $\hat{E}$, the non-uniform branch meets the Rayleigh-Plateau critical point, and at this point it smoothly transits to the non-uniform tube, which has a larger entropy. This transition is not accompanied by a discrete jump in configuration, and therefore is of second or higher order. As $\hat{E}$ decreases further, the non-uniform branch intersects with the ball phase at the point $\mathrm{B}$, and transits to a ball. This transition is of first order.

Thus, the smooth transition from the uniform tube to the non-uniform tube is realized for $d \geq 13$. We conclude that the critical dimension in the microcanonical ensemble is $d_{*}^{\text {microcan }}=13$. Thus, the critical dimension for the gravity dual is expected to be around $D_{*}^{\text {microcan }}=d_{*}^{\text {microcan }}+2=15^{8}$.

\subsection{Canonical Ensemble: $\hat{F}=\hat{F}(\hat{T})$}

In the canonical ensemble, according to the number of cusps appearing in the $(\hat{T}, \hat{F})$ diagram, we again divide the dimensions into three groups $(4 \leq d \leq 9, d=10$, and $d \geq 11)$. The temperature-free energy diagrams for $d=5,10$, and 11 are shown in Fig. 6. Note that as mentioned before, the spherical ball phase has a lower bound of temperature, which is realized when the ball touches itself at the boundary. One can see how the transitions between the three phases occur by repeating discussions similar to those in the previous subsection.

In $d=5$, if we begin with a fat uniform tube (corresponding to a small $\hat{T}$ ) and increase $\hat{T}$, the uniform tube transits to a spherical ball at the point A, which is a first-order transition. The nonuniform tube is not involved in this transition. This transition pattern in the canonical ensemble is similar to that in the Kaluza-Klein black hole-black string system of low dimensions ${ }^{9}$.

\footnotetext{
${ }^{8}$ Here, we adopt the definition of critical dimension by Sorkin [17]. However, if we define a critical dimension, from a non-perturbative viewpoint, by the dimension at and above which the transition from a uniform tube (or uniform black string) to a non-uniform tube (or non-uniform black string) is realized, we find $D_{*}^{\text {microcan }}=13$.

${ }^{9}$ We could not find literature in which the temperature-free energy diagram of the black hole-black string system is presented based on actual numerical data. But, see Fig. 1 in [6] for a temperature-free energy diagram in 10 dimensions speculated from a perturbation analysis, which exhibits a similar behavior to Fig. 6(a).
} 
(a) $d=5$

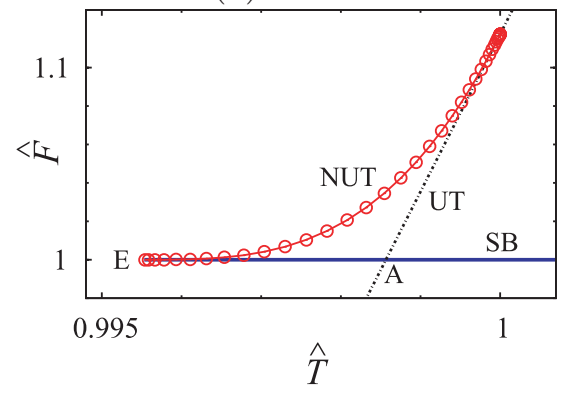

(b) $d=10$

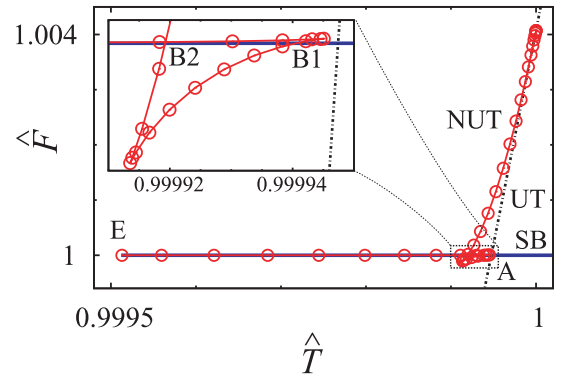

(c) $d=11$

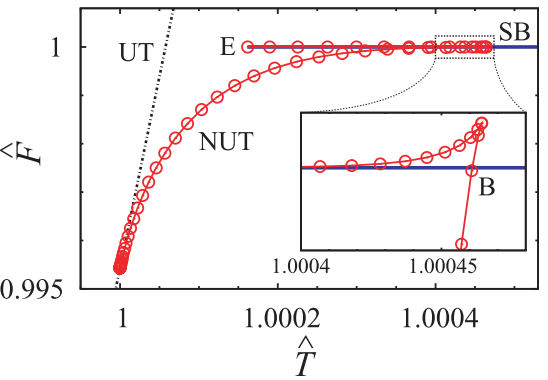

Figure 6: The temperature-free energy diagrams for (a) $d=5$, (b) $d=10$, and (c) $d=11$, containing the phases of spherical ball (SB, blue-solid line), uniform tube (UT, black-dashed curve), and non-uniform tube (NUT, red-solid curve with small circles of data). The phase having the smallest free energy is favored for a given temperature. There is no cusp for $d=5$ (in fact, $4 \leq d \leq 9$ ), while there exist two cusps for $d=10$. One cusp is found in $d=11$ (in fact, $d \geq 11$ ).

The $(\hat{T}, \hat{F})$ curve of the non-uniform tube in $d=10$ has two cusps. However, the non-uniform tube branch is always above the uniform tube branch. Therefore, the non-uniform tube branch has nothing to do with a realistic transition, as the $d=10$ case in the microcanonical ensemble. That is, only the first-order transition from a uniform tube to a spherical ball occurs (at the point A).

In $d=11$, if we begin with a fat uniform tube and increase the temperature, the uniform tube meets the Rayleigh-Plateau critical point, located at $\hat{T}=1$, and at this point it transits smoothly to the non-uniform tube, which has a smaller free energy than the uniform tube phase. This transition is of second or higher order. As the temperature increases further, the non-uniform tube meets a spherical ball at the point B. At this point, it transits to a spherical ball via a first-order transition.

In the canonical ensemble, the critical dimension at and above which the smooth transition from the uniform to non-uniform tube phases is realized is $d_{*}^{\text {can }}=11$. Hence, the critical dimension of the dual black strings is expected to be around $D_{*}^{\text {can }}=d_{*}^{\text {can }}+2=13$.

The patterns of the phase transitions in both microcanonical and canonical ensembles are summarized in Table 1.

Table 1: Diverse patterns of the phase transitions among the uniform tube (UT), non-uniform tube (NUT), and spherical ball (SB) in the microcanonical and canonical ensembles. The ' 2 nd' means that the order of transition may be either second or higher.

\begin{tabular}{|c|c|c|c|c|c|c|}
\hline \multicolumn{2}{|c|}{ Spacetime Dimension on Fluid Side: $d=n+3$} & $4-9$ & 10 & 11 & 12 & $13-\ldots$ \\
\hline \multirow{3}{*}{ Microcanonical } & Transition Type & \multicolumn{2}{|c|}{$\mathrm{UT} \longrightarrow \mathrm{SB}$} & \multicolumn{3}{|c|}{$\mathrm{UT} \longrightarrow \mathrm{NUT} \longrightarrow \mathrm{SB}$} \\
\hline & Order of Transition & \multicolumn{2}{|c|}{1 st } & \multicolumn{2}{|c|}{1 st \& 1st } & '2nd' \& 1st \\
\hline & No. of Cusps in $(\tilde{E}, \tilde{S})$ & 0 & & 2 & & 1 \\
\hline \multirow{3}{*}{ Canonical } & Transition Type & \multicolumn{2}{|c|}{$\mathrm{UT} \longrightarrow \mathrm{SB}$} & \multicolumn{3}{|c|}{$\Longrightarrow \mathrm{UT} \longrightarrow \mathrm{NUT} \longrightarrow \mathrm{SB}$} \\
\hline & Order of Transition & \multicolumn{2}{|c|}{ 1st } & \multicolumn{3}{|c|}{ '2nd' \& 1st } \\
\hline & No. of Cusps in $(\hat{T}, \hat{F})$ & 0 & 2 & \multicolumn{3}{|c|}{1} \\
\hline \multicolumn{2}{|c|}{ Spacetime Dimension on Gravity Side: $D=d+2$} & $6-11$ & 12 & 13 & 14 & $15-\ldots$ \\
\hline
\end{tabular}




\section{Summary and Discussion}

Adopting the equation of state for the fluid obtained by the Scherk-Schwarz compactification of $(d+$ 1)-dimensional conformal field theory, we have investigated the thermodynamic properties of the 'deconfined gluon plasma' lumps, which are expected to be dual to black holes and strings localized in the IR of $\mathrm{AdS}_{d}$ on the Scherk-Schwarz and Kaluza-Klein circles. We have invoked the fluid/gravity correspondence in order to predict the phase diagrams of the AdS black holes and strings. We have found that those phase diagrams are qualitatively similar to those of the black hole-black string system in the asymptotically locally flat Kaluza-Klein space. These results are not surprising in the sense that it had been known that the phase structure of the black hole-black ring system in $\mathrm{AdS}_{5}\left(\mathrm{AdS}_{6}\right)$ with Scherk-Schwarz compactification is qualitatively similar to that of the black hole-black ring system in the 5(6)-dimensional flat background $[4,5]$. However, there is as yet no clear explanation for the agreement between the phase structures of such distinct systems. Furthermore, the critical dimensions found in this paper $\left(D_{*}^{\text {microcan }}=15\right.$ and $D_{*}^{\text {can }}=13$ ) are 'very close' (indeed equal in the canonical ensemble) to the ones in the asymptotically locally flat Kaluza-Klein space $\left(D_{* \mathrm{KK}}^{\text {microcan }}=14\right.$ and $\left.D_{* \mathrm{KK}}^{\mathrm{can}}=13\right)$ in the respective ensembles $[17,18]$. This closeness/coincidence, respectively, may stem from a universality of critical dimension, or there may exist other unknown reasons. As mentioned in the Introduction, the bulk duals may have a non-trivial dependence on the holographic radial coordinate $u$. Furthermore, the distribution of the size of the Scherk-Schwarz circle on horizon cannot be determined a priori before solving the Einstein equations. However, the $u$-dependence as well as the distribution of $\theta$-circles may be simply uniform in the limit of the large black holes/strings for some reason, and in this case the phase structures would resemble those of the Kaluza-Klein system. It would be interesting to clarify the reason for the qualitative agreement, by solving the Einstein equations to find the gravity duals explicitly.

Next, we comment on the validity of our calculation, in particular, under what conditions the effective fluid description of the field theory is valid. One condition is that the length scale over which the temperature and pressure vary must be larger enough than the mean free path of quasiparticles of the system, which is of the same order as the inverse of the deconfinement temperature, $T_{c}^{-1}$ (in the large 't Hooft coupling limit) [1,4]. In our system, the temperature and pressure are constant throughout the configurations, and this condition holds. Another condition is that the temperature must not be far from the critical temperature. Otherwise, it is no longer valid to assume that the surface tension is a constant, whose value was implicitly assumed to be that at the critical temperature $\sigma=\sigma\left(T_{c}\right)$ in this paper. From Fig. 6, we find that the temperature of non-uniform tubes always satisfies $T_{\mathrm{NUT}} / T_{\mathrm{RP}}=O(1)$. Since we have set $T_{\mathrm{RP}} \gtrsim T_{c}$ (by taking $\left.\tilde{l}_{0} \ll 1\right), T_{\mathrm{NUT}} / T_{c}=O(1)$ holds for all configurations near the transition points we are interested in. Finally, the radius of curvature of the fluid surface in all directions must be much larger than the thickness of the surface, which is of order $T_{c}^{-1}$. If this does not hold, higher-derivative contributions to the surface stress tensor must be included. Since we have discussed the case $L \gg l_{0}=\sigma / \rho_{0} \sim T_{c}^{-1}$, we can safely ignore the thickness of the surface. However, the waist of non-uniform tube can become arbitrarily thin in the limit $\lambda=r_{-} / r_{+} \rightarrow 0$, where the thickness of the surface cannot be ignored. Thus, the transition process from a non-uniform tube to a spherical ball could be modified by higher-derivative contributions. One may wonder whether this problem can be resolved by a field theoretic approach.

One may object that the critical dimensions found in this paper are too large to occur in a realistic setting such as M/String theory, and cannot describe reality. However, a critical dimension at and above which a stable non-uniform phase appears will depend on various factors of the system under consideration. For instance, the critical dimension for boosted black strings is expected to 
be significantly lower for highly-boosted strings [19]. Taking into account the fact that a large black ring [20] is regarded as a boosted black string, it is expected that there is a stable undulating black ring phase even in low dimensions such as 5 and 6 . Therefore, it would be quite interesting to investigate the existence of the Rayleigh-Plateau instability for rotating plasma rings [4,5] as well as its possible nonlinear consequences ${ }^{10}$. In a similar vein, it would be also interesting to investigate the RayleighPlateau instability of ultra-spinning plasma balls (see [22] for a related observation on the gravity side), and to examine the (in)stability of the non-uniform tube phases obtained in this paper. These analyses may provide holographic interpretations of the stability of higher dimensional black holes.

\section{Acknowledgments}

The authors would like to thank M.W. Choptuik, O.J.C. Dias, R. Emparan, C. Eling, P. Figueras, G.W. Gibbons, D. Gorbonos, R. Gregory, J. Hansen, T. Harmark, M. Koiso, B. Kol, Y. Mandelbaum, N.A. Obers, B.W. Palmer, H.S. Reall, E. Sorkin, J.H. Traschen, T. Wiseman, and D. Yamada for valuable conversations or comments on this and the related works. U.M. thanks the organizers of the workshop "Black Holes: A Landscape of Theoretical Physics Problems" (25 August - 5 October 2008, CERN), where this work was finalized with the enjoyable discussions with the participants. They would also like to acknowledge hospitality during their stay in September 2008 at DAMTP and the Centre for Theoretical Cosmology, Cambridge University. K.M. is supported in part by the Grant-in-Aid for Scientific Research Fund of the JSPS (No. 19540308) and for the Japan-U.K. Research Cooperative Program, and by the Waseda University Grants for the Special Research Projects. U.M. is supported by the Golda Meir Fellowship, by the Israel Science Foundation Grant (No. 607/05), and by the DIP Grant (No. H.52).

\section{A Manipulations for Accurate Numerics}

The integrands in Eq. (42) diverge since $U\left(w_{ \pm}\right)=0$. Therefore, one needs some manipulations to numerically integrate them accurately, which we describe here.

The variables in Eq. (42), $\tilde{L}(\lambda), \tilde{A}(\lambda)$, and $\tilde{V}(\lambda)$, can be written as

$$
\tilde{X}(\lambda)=\int_{w_{-}}^{w_{+}} \mathrm{d} w \frac{\psi_{X}(w)}{\sqrt{-U(w)}}, \quad(X=L, A, \text { or } V),
$$

where $\psi_{X}(w)$ is a certain regular function, i.e., $\psi_{L}(w):=2, \psi_{A}(w):=2 w^{n} \sqrt{1-U(w)}$, or $\psi_{V}(w):=$ $2 w^{n+1}$. Using the explicit form of the potential $U(w)$, one can write the integral as

$$
\tilde{X}(\lambda)=\int_{w_{-}}^{w_{+}} \mathrm{d} w \frac{\left(w^{n+1}+K\right) \psi_{X}(w)}{\sqrt{\left(w^{n}+w^{n+1}+K\right)\left(w_{+}-w\right)\left(w-w_{-}\right) g(w)}},
$$

where we have defined $(n-1)$-st order polynomial $g(w)$ by

$$
g(w)=\sum_{m=0}^{n-1} g_{m} w^{m}:=\frac{w^{n+1}-w^{n}+K}{\left(w-w_{+}\right)\left(w-w_{-}\right)} .
$$

\footnotetext{
${ }^{10}$ See [21] for an interesting discussion on the (non-)existence of Saturn like configuration of plasma and its implications on the gravity side. We would like to thank one of the authors, C. Krishnan, for informing us of his paper.
} 
Comparing the coefficients of both sides of Eq. (46), we have a recursion relation of $g_{m}$,

$$
\begin{aligned}
& g_{m}-\left(w_{+}+w_{-}\right) g_{m+1}+w_{+} w_{-} g_{m+2}=0, \quad(0 \leq m \leq n-3), \\
& g_{0}=\frac{K}{w_{+} w_{-}}, \quad g_{1}=\frac{\left(w_{+}+w_{-}\right) K}{\left(w_{+} w_{-}\right)^{2}}, \quad g_{n-2}=w_{+}+w_{-}-1, \quad g_{n-1}=1 .
\end{aligned}
$$

This recursion relation is solved as

$$
g_{m}=\frac{g_{n-2}-w_{-} g_{n-1}}{w_{+}-w_{-}} w_{+}^{n-1-m}-\frac{g_{n-2}-w_{+} g_{n-1}}{w_{+}-w_{-}} w_{-}^{n-1-m}, \quad(2 \leq m \leq n-3) .
$$

Using the explicit expressions of $K$ and $w_{ \pm}$, and Eq. (47), we obtain the final expression,

$$
g_{m}=\left(1-\lambda^{m+1}\right)\left(1-\lambda^{n}\right)^{n-2-m}\left(\frac{\lambda}{1-\lambda^{n+1}}\right)^{n-1-m}, \quad(0 \leq m \leq n-1) .
$$

Then, we change the integration variable by

$$
w=\frac{w_{+}+w_{-}}{2}+\frac{w_{+}-w_{-}}{2} \xi
$$

Thus, the integral (44) is written as

$$
\tilde{X}(\lambda)=\int_{-1}^{1} \mathrm{~d} \xi \frac{\left(w^{n+1}+K\right) \psi_{X}(w)}{\sqrt{\left(w^{n}+w^{n+1}+K\right)\left(1-\xi^{2}\right) g(w)}},
$$

where $w$ is regarded as the function of $\xi$ by Eq. (50), and $g(w)$ is given as the polynomial through Eqs. (46) and (49). Finally, we use the following expressions for $\left(K, w_{ \pm}\right)$to reduce the round-off error, which are equivalent to the original definitions (41),

$$
w_{+}=\frac{\sum_{m=0}^{n-1} \lambda^{m}}{\sum_{m=0}^{n} \lambda^{m}}, \quad w_{-}=\frac{\sum_{m=0}^{n-1} \lambda^{m+1}}{\sum_{m=0}^{n} \lambda^{m}}, \quad K=\frac{\left(\sum_{m=0}^{n-1} \lambda^{m+1}\right)^{n}}{\left(\sum_{m=0}^{n} \lambda^{m}\right)^{n+1}} .
$$

We find that the expressions (51) and (52) significantly reduce the numerical errors and make it possible to obtain non-uniform tube solutions, even for $\lambda \ll 1$. A MATHEMATICA notebook is available at the author's website, http://www.phys.huji.ac.il/ umpei/.

\section{B Justification and Interpretation of Cusp Structures}

Here, we argue that the cusp structures observed in the phase diagrams are not numerical artifacts. The phase curves were obtained by numerical integration of Eq. (51), which was done with NIntegrate in the MATHEMATICA code. We confirmed the convergence of integrated values by changing the

Table 2: Numerical values of the slenderness parameter, Eq. (53).

\begin{tabular}{cccccccccccc}
\hline \hline$d=n+3$ & 4 & 5 & 6 & 7 & 8 & 9 & 10 & 11 & 12 & 13 & 14 \\
\hline$\Lambda$ & 3.142 & 2.221 & 1.814 & 1.571 & 1.405 & 1.283 & 1.187 & 1.111 & 1.047 & 0.9935 & 0.9472 \\
\hline \hline
\end{tabular}


parameters of AccuracyGoal, PrecisionGoal, and WorkingPrecision. That is, the digits required to draw the fine structures of phase diagrams such as the cusp structure do not change against the change of these parameters. It is noted that such a convergence is made rapid by virtue of the manipulations described in Appendix A. The fact that the curve of non-uniform tube phase approaches the RayleighPlateau critical point and the tip of the spherical ball phase at the ends of curve supports our numerics independently.

We also note that the existence of cusp structures, especially during the transient dimensions discussed in Secs. 4.1.2 and 4.2, is not inconsistent with our conclusion that the phase structure of black strings and black holes in the Scherk-Schwarz compactified AdS is similar to that of the black hole-black string system in the asymptotically locally flat Kaluza-Klein space. That is, the critical dimensions known in the Kaluza-Klein black hole-black string system $[17,18]$ were predicted by higherorder perturbations, which tells us the behaviors of a phase curve only near the Gregory-Laflamme critical point. Therefore, although the critical dimension of Kaluza-Klein black string has been known to be $D_{*, \mathrm{KK}}=14$ in the microcanonical for example, the possibility is not excluded that the nonuniform black string branches in $D_{\mathrm{KK}}=12$ and $D_{\mathrm{KK}}=13$ behave like the curves of non-uniform tube in Fig. 4 (note that the fully non-linear behaviors of non-uniform black string phase curves have been obtained for $D_{\mathrm{KK}} \leq 11$ in [23], but such a behavior was not observed). Therefore, the cusp structures in the transient dimensions found in this paper are not only consistent with the known gravitational calculations but also suggest an important lesson that a critical dimension should be determined nonperturbatively, or lesson that the critical dimension defined by the smooth transition from the uniform branch to non-uniform branch is not enough to know the global structure of a phase diagram. It would be interesting to perform numerical analyses to obtain the fully non-linear behaviors of non-uniform black strings for $D_{\mathrm{KK}}=12$ and 13, although their difficulty was one of the original motivations to work on fluids in this paper.

In Ref. [11], we found the critical dimension at and above which the non-uniform tube branch emanates from the Rayleigh-Plateau critical point with decreasing volume $V$ for a fixed period $L$, of which existence is related to the critical dimension in the thermodynamic phase diagrams obtained in this paper. We found a simple criterion whether the non-uniform branch increases or decrease its volume near the Rayleigh-Plateau critical point as follows. Let us define a 'slenderness' parameter of the critical uniform tube,

$$
\Lambda:=\frac{L_{\mathrm{RP}}}{2 r_{0}}=\frac{\pi}{\sqrt{n}}
$$

We may say that a critical tube is slender, say, if $\Lambda>1$, while one is fat if $\Lambda<1$. The numerical values of this parameter are given in Table. 2. From the table, the critical tube is found to be slender for $4 \leq d \leq 12$, while fat for $d \geq 13$. On the other hand, the non-uniform tube branch increases its volume for $4 \leq d \leq 12$, while decreases it for $d \geq 13$ near the critical point (see Figs. 2 and 4 in [11]). The above agreement of two threshold dimensions seems not to be just a coincidence from a simple geometric consideration: the non-uniform tube branch emanating from a slender critical tube has to increase its volume for a fixed period $L$ in order to approach a spherical ball, while one emanating from a fat critical tube has to decrease its volume. Thus, the slenderness provides us a simple criterion to forecast the behavior of non-uniform tube around the Rayleigh-Plateau critical point, and our numerical results support this geometric expectation and fit our intuition.

Finally, it should be stressed that the transient behaviors of non-uniform tube observed in Fig. 4 (and also Fig. 6) are quite natural in that the drastic change of phase structure between the lower dimensional class $(4 \leq d \leq 9)$ and higher dimensional class $(d \geq 13)$ was 'smoothly interpolated' by 
the appearance of the 'swallowtail' (i.e., the part of the curve between the pair of two cusps) at $d=10$ and its subsequent growth at $d=11,12$. It would be interesting to regard dimension $d$ as a fictitious continuous parameter and perform the same calculations to obtain phase diagrams for non-integer 'dimensions' $9 \leq d \leq 13$, which would show us a continuous change of phase structure.

\section{References}

[1] D. T. Son and A. O. Starinets, "Viscosity, Black Holes, and Quantum Field Theory," Ann. Rev. Nucl. Part. Sci. 57, 95 (2007) [arXiv:0704.0240 [hep-th]].

[2] S. Bhattacharyya, V. E. Hubeny, S. Minwalla and M. Rangamani, "Nonlinear Fluid Dynamics from Gravity," JHEP 0802, 045 (2008) [arXiv:0712.2456 [hep-th]].

[3] O. Aharony, S. Minwalla and T. Wiseman, "Plasma-balls in large N gauge theories and localized black holes," Class. Quant. Grav. 23, 2171 (2006) [arXiv:hep-th/0507219].

[4] S. Lahiri and S. Minwalla, "Plasmarings as dual black rings," JHEP 0805, 001 (2008) [arXiv:0705.3404 [hep-th]].

[5] S. Bhardwaj and J. Bhattacharya, "Thermodynamics of Plasmaballs and Plasmarings in 3+1 Dimensions," arXiv:0806.1897 [hep-th].

[6] O. Aharony, J. Marsano, S. Minwalla and T. Wiseman, "Black hole - black string phase transitions in thermal 1+1 dimensional supersymmetric Yang-Mills theory on a circle," Class. Quant. Grav. 21, 5169 (2004) [arXiv:hep-th/0406210].

[7] E. Witten, "Anti-de Sitter space, thermal phase transition, and confinement in gauge theories," Adv. Theor. Math. Phys. 2, 505 (1998) [arXiv:hep-th/9803131].

[8] S. W. Hawking and D. N. Page, "Thermodynamics Of Black Holes In Anti-De Sitter Space," Commun. Math. Phys. 87, 577 (1983).

[9] K. Copsey and G. T. Horowitz, "Gravity dual of gauge theory on $\mathrm{S}^{* *} 2 \times \mathrm{S}^{* *} 1 \mathrm{x}$ R," JHEP 0606, 021 (2006) [arXiv:hep-th/0602003]; R. B. Mann, E. Radu and C. Stelea, "Black string solutions with negative cosmological constant," JHEP 0609, 073 (2006) [arXiv:hep-th/0604205]; Y. Brihaye, E. Radu and C. Stelea, "Black strings with negative cosmological constant: Inclusion of electric charge and rotation," Class. Quant. Grav. 24, 4839 (2007) [arXiv:hep-th/0703046]; Y. Brihaye and E. Radu, "Magnetic solutions in $\mathrm{AdS}_{5}$ and trace anomalies," Phys. Lett. B 658, 164 (2008) [arXiv:0706.4378 [hep-th]]; A. H. Chamseddine and W. A. Sabra, "Magnetic strings in five dimensional gauged supergravity theories," Phys. Lett. B 477, 329 (2000) [arXiv:hep-th/9911195]; D. Klemm and W. A. Sabra, "Supersymmetry of black strings in D = 5 gauged supergravities," Phys. Rev. D 62, 024003 (2000) [arXiv:hep-th/0001131]; A. Bernamonti, M. M. Caldarelli, D. Klemm, R. Olea, C. Sieg and E. Zorzan, "Black strings in $A d S_{5}$," JHEP 0801, 061 (2008) [arXiv:0708.2402 [hep-th]]; Y. Brihaye, T. Delsate and E. Radu, "On the stability of AdS black strings," Phys. Lett. B 662, 264 (2008) [arXiv:0710.4034 [hep-th]]; T. Delsate, "Perturbative non uniform black strings in $A d S_{6}$," Phys. Lett. B 663, 118 (2008) [arXiv:0802.1392 [hep-th]]; T. Delsate, "New stable phase of non uniform black strings in $A d S_{d}$," arXiv:0808.2752 [hep-th]. 
[10] V. Cardoso and O. J. C. Dias, "Gregory-Laflamme and Rayleigh-Plateau instabilities," Phys. Rev. Lett. 96, 181601 (2006) [arXiv:hep-th/0602017]; V. Cardoso and L. Gualtieri, "Equilibrium configurations of fluids and their stability in higher dimensions," Class. Quant. Grav. 23, 7151 (2006) [arXiv:hep-th/0610004]; V. Cardoso, O. J. C. Dias and L. Gualtieri, "The return of the membrane paradigm? Black holes and strings in the water tap," Int. J. Mod. Phys. D 17, 505 (2008) [arXiv:0705.2777 [hep-th]].

[11] U. Miyamoto and K. i. Maeda, "Liquid bridges and black strings in higher dimensions," Phys. Lett. B 664, 103 (2008) [arXiv:0803.3037 [hep-th]].

[12] U. Miyamoto, "Curvature driven diffusion, Rayleigh-Plateau, and Gregory-Laflamme," Phys. Rev. D 78, 026001 (2008) [arXiv:0804.1723 [hep-th]].

[13] R. Gregory and R. Laflamme, "Black strings and p-branes are unstable," Phys. Rev. Lett. 70, 2837 (1993) [arXiv:hep-th/9301052]; R. Gregory and R. Laflamme, "The Instability of charged black strings and p-branes," Nucl. Phys. B 428, 399 (1994) [arXiv:hep-th/9404071].

[14] M. M. Caldarelli, O. J. C. Dias, R. Emparan and D. Klemm, "Black Holes as Lumps of Fluid," arXiv:0811.2381 [hep-th].

[15] C. E. Delaunay, J. Math. Pures. Appl. Ser. 1, 6, 309 (1841).

[16] H. Kudoh and T. Wiseman, "Connecting black holes and black strings," Phys. Rev. Lett. 94, 161102 (2005) [arXiv:hep-th/0409111].

[17] E. Sorkin, "A critical dimension in the black-string phase transition," Phys. Rev. Lett. 93, 031601 (2004) [arXiv:hep-th/0402216].

[18] H. Kudoh and U. Miyamoto, "On non-uniform smeared black branes," Class. Quant. Grav. 22, 3853 (2005) [arXiv:hep-th/0506019]; B. Kol and E. Sorkin, "LG (Landau-Ginzburg) in GL (Gregory-Laflamme)," Class. Quant. Grav. 23, 4563 (2006) [arXiv:hep-th/0604015].

[19] J. L. Hovdebo and R. C. Myers, "Black rings, boosted strings and Gregory-Laflamme," Phys. Rev. D 73, 084013 (2006) [arXiv:hep-th/0601079].

[20] R. Emparan and H. S. Reall, "A rotating black ring in five dimensions," Phys. Rev. Lett. 88, 101101 (2002) [arXiv:hep-th/0110260].

[21] J. Evslin and C. Krishnan, "Metastable Black Saturns," JHEP 0809, 003 (2008) [arXiv:0804.4575 [hep-th]].

[22] R. Emparan and R. C. Myers, "Instability of ultra-spinning black holes," JHEP 0309, 025 (2003) [arXiv:hep-th/0308056]; R. Emparan, T. Harmark, V. Niarchos, N. A. Obers and M. J. Rodriguez, "The Phase Structure of Higher-Dimensional Black Rings and Black Holes," JHEP 0710, 110 (2007) [arXiv:0708.2181 [hep-th]].

[23] E. Sorkin, "Nonuniform black strings in various dimensions," Phys. Rev. D 74, 104027 (2006) [arXiv:gr-qc/0608115]. 